\title{
Asesoramiento a equipos de gestión y liderazgo educativo desde el abordaje de las competencias funcionales ${ }^{1}$
}

\author{
Carolina Iturra Herrera ${ }^{\text {a }}$ \\ Víctor Eduardo Cancino Cancino b
}

\section{Resumen}

Este trabajo presenta una experiencia de asesoramiento a equipos directivos de la región del Maule, Chile, enfocado principalmente en el aprendizaje de competencias funcionales asociadas a los desempeños de los equipos directivos. Para ello, se diseñó, ejecutó y evaluó un programa de acompañamiento socioeducativo en el que participaron 11 establecimientos educacionales. Los resultados analizados de acuerdo a dos niveles de evaluación de logros de aprendizaje mostraron un alto nivel de satisfacción de los participantes, así como aprendizajes relacionados con los pasos para la solución de problemas.

Palabras clave: Liderazgo educativo. Programa asesoramiento. Competencias funcionales.

\section{Introducción}

Se reconoce que el desempeño y el tipo de liderazgo que se ejercen en las instituciones educativas es uno de los factores claves que permitiría mejorar los logros de aprendizaje. En este sentido, los resultados de diversas investigaciones internacionales (LEITHWOOD et al., 2006) indican que el tipo de liderazgo ejercido en las instituciones educativas sería la segunda variable intraescolar que influiría en la capacidad y logros de aprendizaje de los estudiantes en las aulas.

Para que éste liderazgo promueva aprendizajes de calidad en las escuelas es necesario introducir modificaciones a los desempeños de los equipos de gestión. Ello implicaría un cambio en la función desempeñada por los directores o equipos de gestión de los

\footnotetext{
El presente trabajo fue realizado gracias al Convenio de Desempeño en Humanidades, Artes y Ciencias Sociales realizado entre la Universidad de Talca y el Ministerio de Educación de Chile (TAL901).

a Universidad de Talca. Talca, Chile.

b Universidad Santo Tomás. Santiago, Chile.
} 
establecimientos educacionales, transitando desde una mirada centrada en tareas y actividades burocráticas clásicas hacia otros paradigmas orientados por los liderazgos educativos con foco en la mejora de los procesos internos de las escuelas (BOLÍVAR, 2010) que posibiliten y aseguren el proceso de aprendizaje de calidad para todos los estudiantes de los centros educativos (DARLING-HAMMOND, 2001).

$\mathrm{Al}$ respecto, este cambio de paradigma implicaría posicionar a los directores de establecimientos y sus equipos directivos como aquellos líderes que deben orientar el curso de acciones, estableciendo metas vinculadas a los resultados educativos y desarrollar planes para promover mejoras en el quehacer docente. Para ello, se hace indispensable que los directores y sus equipos de gestión modifiquen sus prácticas, implicando ello aprender una serie de conocimientos, procedimientos, hábitos y habilidades (ELMORE, 2010) que contribuyan al mejoramiento de la calidad escolar.

Bajo esta mirada, tanto a nivel internacional como en Chile, se ha puesto de relieve la necesidad de asesorar y apoyar la enseñanza y desarrollo profesional de los directores y equipos de gestión para favorecer esta transición y mejorar el ejercicio de las direcciones centrándose en los resultados de aprendizaje y en prácticas que crean contextos para la apropiada acción pedagógica en las aulas (WEISTEIN, 2009).

En este marco, el presente trabajo busca describir una experiencia de asesoramiento a los directores y equipos de gestión de distintos establecimientos educativos de la región del Maule, Chile, al alero de un proyecto de Convenio de Desempeño de la Universidad de Talca denominado "Desarrollo y Fortalecimiento de las Humanidades, Artes y Ciencias Sociales, en su Vinculación con el Medio Regional: Una Propuesta desde el Valle Central.", que dentro de sus objetivos se encontraba el desarrollo de un programa de intervención multidisciplinaria en comunidades escolares de la Región. Dentro de las distintas acciones enmarcadas, la Facultad de Psicología de la Universidad de Talca diseñó y ejecutó un programa de acompañamiento y asesoramientos a competencias funcionales socioeducativas de equipos directivos.

\section{Marco contextual}

\subsection{Prácticas de liderazgo efectivas}

Baldrige (National Institute of Standards and Technology, 1995) define la eficacia directiva sustentada desde modelos de gestión de la calidad, explicando que ésta es lograda mediante la acción colectiva de un líder y su equipo, quienes son los encargados del diseño de políticas, procedimientos, directrices, prácticas 
instruccionales y supervisión de toda la escuela. En la misma línea, Leithwood (2009) propone que los liderazgos efectivos desplegarían un conjunto de prácticas constantes que se adecuarían a los contextos dependiendo del tipo de estudiantes, el tipo de establecimiento y la etapa o grado de avance en su mejoramiento y que se relacionarían con acciones orientadas hacia la motivación de las personas para realizar sus trabajos; planificación de un conjunto de acciones para el desarrollo profesional de las personas a su cargo; establecimiento de climas y condiciones que permitan un rediseño de la organizaciones y supervisión de las prácticas pedagógicas en las aulas. Asimismo, Leithwood, Harris y Hopkins (2008), precisan los dominios o dimensiones de la actuación de directivos inciden en el colectivo de docentes y a nivel individual, señalando que los directores escolares influencian fuertemente las condiciones laborales y la motivación de los docentes, impactando indirectamente en el aprendizaje de los estudiantes.

A nivel internacional y a partir de la experiencia de los países de la OCDE, Pont, Nusche y Moorman (2008) presentan ciertas recomendaciones para promover políticas educativas y prácticas liderazgo en directivos escolares relacionadas con la necesidad de fortalecer la práctica de dirección escolar, entendiendo que este liderazgo es un continuo que aludiría a trayectorias de desarrollo y por tanto, debiera acompañarse de políticas que otorguen incentivos y que favorezcan programas de inducción y educación continua de calidad. Estas últimas debieran responder a las demandas de contenidos y habilidades requeridas por los líderes, ser periódicas y fortalecer el aprendizaje de conocimientos y competencias que propicien el trabajo en equipo.

En este marco, el Ministerio de Educación Chileno ha generado como línea estratégica el fortalecimiento de liderazgos educativos en los establecimientos educacionales. Dentro de algunas iniciativas recientes se encuentran la construcción del Marco para la Buena Dirección, cuya primera versión estableció un conjunto de estándares de desempeño que buscaba orientar las acciones de los directores, además de promover su desarrollo profesional. Este marco de actuación fue actualizado en 2015 elaborándose una versión que profundiza en las competencias $\mathrm{y}$ habilidades asociadas a la función directiva y para el ejercicio de un liderazgo escolar contextual y contingente (CHILE, 2015).

Por otra parte, en el año 2011 se implementó la Ley n 20.501 (CHILE. 2011) sobre calidad y equidad de la educación dando inicio a cambios relevantes en la gestión directiva, destacando la implementación del Sistema de Alta Dirección Pública que busca modernizar y regular la concursabilidad para asumir la función directiva escolar, además de conferirle a los directores mayores atribuciones para 
la gestión de los establecimientos, en materia de recursos humanos para organizar, supervisar y evaluar el trabajo de los docentes y del personal administrativo, así como mayores garantías y mejoras salariales asociadas al cumplimiento de metas (CANCINO; VERA, 2017).

Concordante con esta acciones, se define un plan de formación que cuenta con objetivos de aprendizaje explícitos (CHILE, 2013) que buscarían que los directores adquirieran competencias que les permitieran establecer direcciones mediante la planificación de acciones a largo plazo, diseñando estrategias para alcanzar las metas propuestas, competencias asociadas al desarrollo de personas mediante la construcción de ambientes positivos para el apoyo pedagógico, competencias para el rediseño de las organizaciones escolares promocionando culturas orientadas a la calidad y al desarrollo profesional docente y competencias para gestionar los programas de enseñanza gestionando recursos humanos y pedagógico para asegurar los aprendizajes en las aulas.

Estas distintas iniciativas ministeriales han permitido el desarrollo de investigaciones orientadas a describir el contexto de liderazgo educativo en Chile, además de la elaboración de propuestas para programas de formación. Dentro de ellas, el trabajo de Muñoz Stuardo y Marfán Sánchez (2011) ha diferenciado dos tipos de competencias vinculadas a las prácticas efectivas de los directores y equipos de gestión. Las primeras denominadas competencias conductuales se relacionarían con elementos transversales y actitudinales que sustentarían el trabajo del director e incluirían las disposiciones previas de los mismos, mientras que las competencias funcionales harían alusión a la capacidad para desempeñar acciones específicas de las funciones propias de los cargos directivos.

Respecto a las competencias funcionales, éstas se agruparían en cinco dimensiones. En primer lugar, "Establecer una visión orientadora" se relacionaría con la capacidad del director para orientar acciones de la comunidad escolar vinculadas con los proyectos educativos y dirigidas a la mejora de los logros de aprendizaje a través pasos y herramientas factibles de llevar a cabo en el establecimiento, promocionando un adecuado nivel de expectativas. La segunda dimensión "Generar condiciones organizacionales" implicaría una apropiada gestión de recursos y funciones, así como el establecimiento de redes de apoyo dirigidos al cumplimiento del proyecto educativo. La tercera dimensión "Gestión de la convivencia escolar" permitiría desplegar competencias orientadas a la gestión de las relaciones en la comunidad escolar y la promoción de ambientes seguros. La cuarta dimensión "Desarrollar personas" se vincularía con las capacidades de los equipos de gestión para 
delinear adecuadas trayectorias de desarrollo profesional de los profesores y finalmente la quinta dimensión "Gestionar la pedagogía" se traduciría en las competencias directivas para el diseño de acciones y mecanismos técnicopedagógicos para apoyar y asesorar un adecuado desempeño de los profesores en su quehacer docente.

Es desde esta línea centrada en las competencias funcionales que el equipo de diseño y ejecución del programa de asesoramiento y acompañamiento, orientó el trabajo con los directores y equipos de gestión.

\subsection{Aprendizaje de los profesores y solución de problemas}

Tal y como señalábamos en los párrafos precedentes, se han iniciado procesos que buscan modernizar el desempeño de los directores y equipos directivos, orientándolos fundamentalmente a la gestión de acciones estratégicas que permitan mejorar la calidad y los logros de aprendizaje de los estudiantes. En este sentido, es importante señalar que la forma en cómo se diseñan y ejecutan estos programas de enseñanza de las competencias asociadas al desempeño directivo son fundamentales. Por tanto, se hace necesario que la configuración de los procesos de enseñanza-aprendizaje para los directores integren una serie de principios o líneas de trabajo metodológicas que permitan lograr un aprendizaje situado. Desde esta línea, hoy en día se reconoce que los aprendizajes se negocian y construyen en interacción social y que son facilitados cuando se insertan en los escenarios donde se aplicarán dichos conocimientos (BORKO, 2004).

$\mathrm{Al}$ respecto, la literatura internacional señala que parte del desafío para lograr poner en acción los procesos de reformas implican que los participantes, en este caso, los directores y equipos de gestión, deben participar de espacios que les permitan la reflexión sobre su práctica y experiencia. Desde esta mirada, Putnam y Borko (2000) proponen que los programas de formación eficaces deberían incluir ciertas directrices necesarias para generar estos escenarios de innovación y cambio. Estas directrices se relacionarían con la necesidad de definir la figura de los profesores/directores como sujetos que aprenden activamente y construyen sus propias interpretaciones y por tanto, se requeriría reconocer su dominio profesional.

Por otra parte, se plantea que la formación debe diseñarse a través de experiencias prácticas y desde la práctica, promoviendo tareas auténticas que les permitan razonar y resolver problemas. En esta misma línea, algunos trabajos nacionales (LABRA et al., 2005; MONTECINOS; CORTEZ, 2015) plantean la necesidad de que éstas experiencias prácticas permitan reflexionar no sólo sobre 
las acciones, sino también desde aquellos conocimientos tácitos e identidades profesionales que permitirían entender dichas acciones. Particularmente el trabajo de Montecinos y Cortez (2015) definen que los aprendizajes situados en la enseñanza y desarrollo de capacidades de liderazgo, deberían promover la construcción o reconstrucción de los elementos que subyacen a las prácticas o patrones que despliegan los directores incluyendo conjuntamente nuevas configuraciones o patrones sociales y materiales. Asimismo, orientarse hacia el desarrollo profesional en todos sus aspectos de manera integral y así como a la solución de problemas y toma de decisiones frente a dificultades prototípicas del quehacer directivo, enmarcados en contextos de aprendizaje colaborativo y distribuido.

Considerando estos lineamientos, uno de los aspectos importantes de potenciar en la formación de los directores y equipos de gestión directiva, es el enfrentamiento a problemas para mejorar su abordaje y solución. Al respecto Leithwood y Stager (1989) plantean que el proceso de solución de problemas es fundamental para la toma de decisiones relacionadas con los planes y acciones que conducen el quehacer de las escuelas. Señala que los liderazgos escolares se enfrentan a un sin número de problemáticas en su práctica cotidiana, muchas de las cuales son de difícil resolución, dado que obedecerían a problemas mal estructurados, complejos y multidimensionales. Estos problemas propios de las ciencias sociales, se constituirían de variados factores y causas de distinta entidad, individual y colectiva que exigen diferenciar entre premisas y conclusiones e implican acciones que con una secuenciación histórica y temporal. Frente a esto, Leithwood y Stager (1989) definen una serie de componentes que permitirían categorizar los juicios emitidos por directores, frente a los pasos implicados en el proceso de solución de problemas. Estos componentes serían: Interpretación definido como el grado de entendimiento sobre la naturaleza del problema o problemas. Objetivos que harían alusión a las metas inmediatas para tratar de resolver el problema interpretado. Principios, consistirían en las metas a largo plazo, directrices y supuestos que orientan el pensamiento de director. Barreras, entendidas como aquellas amenazas que limitarían la puesta en práctica de las acciones para resolver el problema. Procesos serían los planes y las acciones para resolver el problema.

En síntesis y considerando la necesidad de fortalecer los liderazgos educativos de los directores y equipos de gestión de los establecimiento educacionales mediante la potenciación y trabajo en competencias que les confieran capacidades y habilidades para orientar el quehacer en las escuelas, se diseñó, ejecutó y evaluó una experiencia de acompañamiento a directores y equipos de gestión, que pasamos a detallar a continuación. 


\section{La experiencia}

\subsection{El diseño de intervención}

En el marco de la implementación y ejecución del Convenio de Desempeño en Humanidades, Música y Ciencias Sociales celebrado entre la Universidad de Talca y el Ministerio de Educación Chileno, la Facultad de Psicología diseñó un programa de asesoramiento e intervención psicosocial para establecimientos educacionales de la Región del Maule, Chile. El foco particular de esta intervención consistió en el fortalecimiento de competencias funcionales socioeducativas de equipos directivos, entendiendo estas competencias como las capacidades específicas para desarrollar acciones vinculadas directamente a las tareas y funciones de la dirección de establecimientos educacionales (URIBE, 2010). Dentro de estas competencias se distinguen aquellas que permitirían una visión orientada hacia el logro de aprendizajes escolares y la gestión de la pedagogía, capacidades para la gestión de personas y condiciones organizacionales y competencias para la gestión de la convivencia escolar (MUÑOZ STUARDO; MARFÁN SÁNCHEZ, 2011).

Por tanto, se definió la intervención como un conjunto de acciones orientadas al logro de objetivos y aprendizajes que permitieran potenciar habilidades y competencias asociadas al desempeño de funciones propias del cargo. Para ello, se definieron etapas claves que guiarían el proceso de asesoramiento, siendo estas un diagnóstico en cada establecimiento, la puesta en marcha del proceso de acompañamiento e intervención y la evaluación de la misma.

\subsubsection{Caracterización grupo objetivo}

Tal y como se describe en la Tabla, se incluyeron dentro de los establecimientos participantes, tres de carácter subvencionado, todos de tipo confesional, es decir, colegios que poseen una administración privada pero financiamiento a través de subvención por estudiante matriculado, mientras que se incluyeron 8 establecimientos municipales administrados por las municipalidades que reciben subvención directa y única a través del Estado. El criterio de inclusión de los

Tabla. Caracterización de los participantes según año del programa y tipo de institución.

\begin{tabular}{lccc}
\hline & $\begin{array}{c}\text { Colegios } \\
\text { Subvencionados }\end{array}$ & $\begin{array}{c}\text { Colegios } \\
\text { Municipales }\end{array}$ & $\begin{array}{c}\text { Total por } \\
\text { Año }\end{array}$ \\
\hline Año $2014 n^{\circ}$ colegios & 3 & 3 & 6 \\
Año $2015 n^{\circ}$ colegios & & 5 & 5 \\
\hline
\end{tabular}

Fuente: Elaboración própria (2017). 
colegios participantes fue el índice de vulnerabilidad de la población atendida, que en este caso fue de un nivel medio-bajo y bajo.

En el diagnóstico se aplicaron entrevistas semi-estructuradas, recolectando información e indicadores respecto a los procesos organizacionales y las principales fortalezas y debilidades, además de análisis documental de sus proyectos educativos, planes de mejoramiento, organigramas e información en línea de los establecimientos. Las áreas diagnosticadas permitieron precisar en cada colegio, las acciones relacionadas con la gestión de resultados y el trabajo desarrollado por los directivos, además de identificar las redes de apoyo institucionales y el desarrollo de planes para la gestión de la convivencia escolar.

Los resultados confirieron al equipo de asesoramiento, la posibilidad de obtener información para ser trabajada en cada sesión de intervención. Por otra parte, se identificaron niveles distintos de afrontamiento de las situaciones problema y de los recursos para la toma de decisiones.

\subsection{Intervención}

El foco se centró particularmente en potenciar la capacidad de los integrantes de los equipos de gestión para utilizar los resultados de aprendizaje para la toma de decisiones, la capacidad para la elaboración de programas de mejoramiento y acciones para el monitoreo de la práctica docente. Para ello, una de las orientaciones asumidas por el equipo, fue potenciar capacidades de resolución de problemas intencionando el aprendizaje para que los participantes identificaran las dificultades o problemas, los analizaran y desarrollaran acciones alternativas para remediarlos. En este sentido, el equipo de diseño de la intervención adscribió a la naturaleza del aprendizaje situado (BORKO, 2004), entendiendo la necesidad de incluir en la metodología, tareas auténticas vinculadas a la realidad de cada equipo de gestión, que contribuyera a la identificación de problemáticas y la construcción de acciones de mejoramiento, pero además, a la creación de comunidades de aprendizaje (MARCELO, 2002) que permitieran a los participantes del taller, relacionarse con otras realidades y potenciar sus visiones, incluyendo experiencias y prácticas distintas.

Las líneas temáticas revisadas a lo largo de las sesiones fueron diagnóstico de la institución, gestión del Clima Organizacional y Convivencia Escolar y gestión de resultados pedagógicos. Cada sesión tuvo una duración de 6 horas y en total se desarrollaron 5 sesiones en dependencias de la Facultad de Psicología de la Universidad de Talca. 
Desde el punto de vista metodológico, cada una de las sesiones se organizó en función de actividades estratégicas que implicaban una hora de exposición teórica a cargo de un profesor invitado o un asesor con experticia en el tema trabajado. Posteriormente, se desarrollaban talleres de aplicación mediante análisis de casos o detección de problemáticas propias de cada colegio.

\subsection{Evaluación de la intervención}

El propósito de la evaluación del programa consistió en contar con información que retroalimentara el diseño de la intervención. Para ello, se utilizó como marco de referencia la adaptación del modelo de Kirkpatrick's para la evaluación de logros de aprendizaje y programas de educación superior (PRASLOVA, 2010). En esta adaptación que incluye los cuatro niveles propuesto por Kirkpatrik's (Reacción, aprendizaje, conducta y resultado), definiéndose para cada uno de ellos, los criterios incluidos en cada nivel y algunos ejemplos de instrumentos. Así, el nivel reacción buscaría describir reacciones afectivas respecto a los procesos formativos y juicios de utilidad vinculados con la percepción respecto a lo aprendido. El segundo nivel aprendizaje se orientaría a precisar el nivel de conocimiento alcanzado, a través de mediciones de los rendimientos, para ello, existirían una serie de instrumentos desde los más tradicionales como pruebas o algunos más innovadores como resolución de casos o ensayos. El tercer nivel también es definido como criterios de comportamiento o transferencia e incluyen medidas de rendimiento en los contextos laborales o de práctica en los que es posible observar el desempeño adquirido durante los procesos formativos. Finalmente, el cuarto nivel, resultados implicaría el impacto o efecto del proceso formativo en las instituciones u organizaciones en las que los aprendices se desempeñarían, sin duda, este nivel sería complejo de medir, dado que incluiría una serie de variables o dimensiones, algunas de ellas ajenas al proceso formativo.

Se debe precisar que dado el alcance del asesoramiento e intervención, tanto en términos temporales como de profundización del trabajo, sólo se logró diseñar instrumentos de evaluación para los dos primeros niveles.

\subsubsection{Nivel reacción}

El objetivo en este nivel consistió en identificar la percepción de los participantes respecto al taller, desde el punto de vista de sus apreciaciones afectivas vinculadas con la motivación y la metodología experimentada, como su percepción respecto a la utilidad del mismo. Para ello, se utilizó como instrumento de recogida de información la técnica de focus group. Dentro del guión temático se abordaron 
tópicos vinculados con la apreciación respecto al diseño y ejecución del programa, evaluación de lo aprendido y su transferencia a los contextos laborales, motivación y sugerencias de mejora. Como procedimiento de análisis, se aplicó análisis de contenido (RUIZ OLABUÉNAGA, 2012) estableciendo niveles de categoría previamente vinculadas con las temáticas abordadas. En relación a los participantes, un total de 7 personas se incluyeron, representando a distintos tipos de establecimientos.

Dentro de los criterios de veracidad (LINCOLN; GUBA, 1985) incluidos en este nivel, se pueden mencionar la credibilidad y dependencia, dado que el desarrollo del focus, así como el primer análisis del mismo fueron desarrollados por un psicólogo externo al grupo de trabajo, además las sesiones fueron grabadas con autorización de los participantes, para recurrir constantemente a los datos para interpretarlos, asimismo, se utilizó triangulación de criterios entre el equipo de trabajo y el psicólogo externo. Respecto a la tranferibilidad, se utilizaron criterios opináticos o de convivencia de la muestra que permitieron que esta fuera representativa.

\subsubsection{Nivel aprendizaje}

El objetivo de este nivel fue identificar el grado de conocimiento alcanzado por los participantes de los talleres, respecto a los pasos de las líneas estratégicas trabajadas en cada una de las sesiones. Se diseñaron casos como instrumentos de recolección de información que implicaban para los participantes definir un plan de acción. Posteriormente, se realizó una entrevista grupal para ahondar respecto al procedimiento utilizado por cada equipo en la resolución de los casos. El análisis de la información recabada se realizó mediante un análisis de contenido que contó con cinco categorías a-priori: Interpretación, objetivos, principios, metas y procesos. Respecto a los casos, el primero consistió en un establecimiento educacional con un grupo de profesores que dificultades para la construcción de ambientes de aprendizaje propicios para el mismo. El segundo caso se situó en un establecimiento educativo que atendía población vulnerable y que presentaba dificultades en las tasas de asistencia de los estudiantes acompañado de inadecuados resultados educativos, además de identificarse poco compromiso de parte de los profesores y dificultades en el cumplimiento del rol de profesor jefe. Participaron un total de 9 personas organizadas en 2 equipos distintos. Respecto a los criterios de veracidad, nuevamente se recurrió a un asesor externo quien apoyó en el diseño de casos y dirigió la aplicación de los mismos, la discusión en la entrevista grupal y el análisis, logrando de esta manera cumplir con los criterios de credibilidad y dependencia. 


\section{Resultados}

\subsection{Nivel Reacción}

A continuación, se exponen los principales resultados emanados del análisis del focus group.

\subsubsection{Reacciones afectivas}

Los participantes manifestaron que durante la ejecución de los talleres sintieron un ambiente grato de trabajo en que fueron atendidas la mayoría de sus ideas y problemáticas. Destacaron que el clima de trabajo potenció la interacción entre los participantes, integrando aspectos de las realidades de los distintos centros educacionales y de las experiencias de personales y profesionales de los participantes. En ese sentido valoraron positivamente la participación de otros centros, debido a que el intercambio de experiencias y apreciaciones generó una dinámica constructiva y enriquecedora para los participantes.

muchas veces nos tocó trabajar como grupo, con otros colegios y muchas veces como roles de cada uno de otros inspectores donde compartíamos la experiencia, teníamos que trabajar en grupo, y ninguno era más que el otro, éramos todos iguales, directores con directores, orientadores con orientadores.

Por otra parte, sostuvieron que se cumplieron sus expectativas respecto al programa, valorando la calidad de éste y de los distintos expositores, además de sentirse motivados a participar activamente. Todos los participantes refirieron que las herramientas trabajadas les aportaron para tener una visión más amplia del quehacer de sus roles dentro de los equipos directivos, permitiéndoles la construcción de una visión menos crítica de sus desempeños menos crítica de ellos mismos.

lo primero es que es un regalo, hoy días las cosas cuestan mucho y considero que esto si tuviese que pagarlo, tendría un alto costo, hoy día los recursos son escasos instituciones no andan regalando cosas, entonces yo eso lo valoro muchísimo, por eso nuestra asistencia era permanente, excepto que le pasara algo a uno de los cuatro pero era asistencia permanente, porque recibir un regalo es nada más que para esta contento, agradecerlo, eso es lo primero. 


\subsubsection{Juicios de utilidad}

Los participantes refirieron que los contenidos trabajados fueron atingentes a sus realidades educativas. Por otra parte, sostuvieron que la metodología utilizada fue adecuada y que permitió la integración entre los contenidos trabajados y su proceso de aprendizaje. Destacaron la metodología utilizada, que les permitió aplicar las herramientas aprendidas en sus contextos educativos. Dentro de los aspectos que mencionaron haber aprendido y transferido a sus realidades, destacaron herramientas de planificación de proyectos, evaluación, herramientas comunicacionales y trabajo en equipo para mejorar relaciones interpersonales y comunicacionales, herramientas de planificación y seguimiento de resultados de aprendizaje, como implementación de recursos y estrategias de mejoramiento de la práctica educativa en el aula.

yo asistí a todos los talleres del equipo directivo y si creo que se renuevan conocimientos se renuevan conocimientos, se generan algunas competencias que en algunos casos estaban medias o no las teníamos desarrolladas, trabajamos en concreto con algunas acciones de nuestro PME, del año que teníamos que estructurar, modificar, así que trabajamos bien en concreto...

En síntesis, tras el análisis se constató que las percepciones explicitadas por los participantes del focus group valoraron el programa positivamente, dado que este aportó herramientas concretas para utilizarlas en sus realidades educativas y posibilitó el conocimiento e interacción con otras, lo que favoreció la integración y modificación de sus propias visiones acerca de su propio desempeño.

\subsection{Nivel aprendizaje}

En este nivel se presentarán los análisis en relación a cinco categorías: Interpretación, objetivos, principios, barreras y procesos.

\subsubsection{Interpretación}

En ambos casos, señalaron que las dificultades se relacionarían con el desempeño de los profesores y que este hecho alteraría el funcionamiento adecuado en las dimensiones pedagógicas y de convivencia.

El equipo 1 manifestó que las principales dificultades consistirían en inadecuados ambientes de aprendizaje que impactaría negativamente en los resultados de aprendizajes. En este sentido, señalaron que el foco del problema radicaría en que estos profesores no tendrían las herramientas necesarias para un adecuado 
manejo en las aulas, conjuntamente con dificultades para planificar y estructurar cada una de las sesiones.

A nosotros nos tocó que había un porcentaje de docentes en nuestros establecimientos que hemos identificado que no hace énfasis en normalizar el ambiente de la clase y eso genera problemas con los aprendizajes evidentemente porque puede tenderse a preocuparse por el interés de un grupo y el resto del grupo hace lo que puede y pierde la posibilidad de aprender.

En el equipo 2 definieron que las principales dificultades se centrarían en problemáticas vinculadas por un lado, con el rol de profesor jefe y su relación con el proyecto educativo institucional y los altos niveles de inasistencia y desmotivación de los estudiantes, delimitando que se centrarían en el desempeño de los profesores jefes.

Nosotros tenemos tres problemas: el primero es el cambio de perfil del rol del profesor jefe, de acuerdo al proyecto educativo institucional. El segundo es la pérdida de identidad con la institución por parte de los docentes, y eso réplica en los estudiantes, y el tercer problema era los altos niveles de inasistencia de los estudiantes, desmotivados por la ausencia de docentes.

\subsubsection{Objetivos}

En esta categoría, ambos equipos señalaron la necesidad de aplicar procesos de diagnóstico que les permitiera objetivar la realidad y contar con información para una adecuada toma de decisiones. También en ambos equipo señalaron la necesidad de utilizar instrumentos para la recolección de información, identificándose diferencias de acuerdo a los casos analizados.

\section{Equipo 1}

Entonces primero hay que diagnosticar, y para eso necesitaríamos más de una lectura para ser certeros en identificar esos profesores. Una cosa podría ser una conversación entre el directivo que conoce al profesor, que damos clases y conocemos y vemos la realidad, lo otro podría ser hacer una lista de cotejos de algunas acciones que se ven o no se ven de lo que él hace en sus clases entonces así podríamos aplicar directamente a la mayoría y darnos cuenta por dónde irían las necesidades. 


\subsubsection{Principios}

En esta categoría, los dos equipos lograron explicitar una visión orientadora relacionada con el mejoramiento de los resultados de aprendizaje, implicando metas a largo plazo que se deberían promover como parte de acciones estratégicas, además de identificar los distintos cargos dentro de los equipos directivos que debieran estar incluidos en dichas acciones.

El equipo 1 manifestó la necesidad de contar con un sistema de acompañamiento constante a los profesores, para monitorear y apoyar su desempeño en las aulas.

Entonces desde esa perspectiva yo siento que tiene que hacer una apertura tanto en la unidad educativa para que se fortalezca este proceso de mejoramiento porque si yo lo voy a mirar desde punto de vista negativo y hacia los profesores debe haber un proceso de sensibilización en donde se releve la importancia que tiene para nosotros el mejoramiento de la práctica.

Por otra parte, el equipo 2 explicitó metas relacionadas con la necesidad de lograr una mayor coherencia entre el proyecto educativo vigente y el desempeño de los profesores jefes como una estrategia orientada a la mejora de los aprendizajes escolares, además de diseñar mecanismos que permitan monitorear constantemente esta coherencia.

Mirando el proyecto educativo institucional Y puede ser cada semestre a lo mejor hacer una encuesta a los apoderados, una encuesta a los estudiantes, para ver cómo ha ido el desempeño" "queremos establecer lineamientos comunes a través de documentos que orienten la labor del profesor jefe. Los responsables en ambos casos serían el equipo directivo.

\subsubsection{Barreras}

Respecto a esta categoría, ambos equipos identificaron ciertas amenazas que podrían limitar las distintas acciones planificadas. En algunos casos, estas barreras aludieron a causas extraescolares, mientras que otras se relacionaban directamente con causas intraescolares.

En el caso del equipo 1, estas barreras se vinculaban a las dificultades que implica trabajar y liderar a personas. 
cuando uno toma un profesor par, para darle una función de relieve, muchos dicen por qué a mí no, no tienen la humildad a lo mejor para reconocer si tenía las habilidades... entonces eso genera a veces un anticuerpo, nosotros decíamos que a estos colegas hay que darles un apoyo especial y hay que saber también cómo comunicar a los demás colegas, qué es lo que esperamos, y que es un soporte de apoyo más que ponerle conflictos a la temática.

Por otra parte, el equipo 2, además de mencionar barreras intraescolares como la valoración del cargo de profesor jefe, manifestó que en algunas ocasiones, los profesores no cuentan con el conocimiento ni las competencias desde el egreso, para poder desempeñar funciones asociadas al cargo.

Ahí es que uno se hace la pregunta... todo profesor que está recién egresado, debería tener o desarrollar habilidades para el manejo de personas... Porque tú a veces ves a un profesor que a nivel disciplinario puede manejarse muy bien pero cuando se trata de orientar a los alumnos no tiene idea...

\subsubsection{Procesos}

Finalmente, en la categoría procesos, ambos equipos identificaron metas a corto plazo, establecieron planes de acción y mecanismos de monitoreo para resolver los problemas planteados.

Equipo 1

realizar acompañamiento mediante mentores, hacia estos colegas que tienen esa debilidad, de normalización en el aula, nosotros pretendemos que el ciento por ciento de los colegas que y que fueron identificados, hagan una mejora respecto al diagnóstico que tuvimos, y hagan una mejora en la normalización en sus clases.

\section{Conclusiones}

Como se señaló al principio del artículo, el principal objetivo de este consistía en describir una experiencia orientada a fortalecer el quehacer de equipos directivos, mediante un programa de acompañamiento en las que se potenciaron competencias funcionales. En este sentido, se debe señalar que el alcance del programa no logró la cobertura ni la profundización necesaria para trabajar todas las competencias funcionales necesarias, como tampoco se alcanzó a evaluar todos los niveles para 
constatar cual es la mejor alternativa, no obstante, si permite corroborar ciertos principios necesarios para modernizar y apoyar los procesos de cambio que se requieren en pos de alcanzar una mayor calidad en los resultados de aprendizaje de los estudiantes.

En primer lugar, se considera fundamental contar con marcos regulatorios claros y acotados para orientar el diseño de programas que pretendan desarrollar o fortalecer competencias en equipos directivos. Desde esta mirada, el propio programa incluyó los lineamientos ministeriales en relación a las metas o estándares asociados por ejemplo, a al Marco para la Buena Dirección (CHILE, 2015) o los propios objetivos de aprendizaje definidos para la formación de excelencia de directores (CHILE, 2013). Sin embargo, se debe señalar que estos marcos requerirán de ajustes constantes en la medida en que las demandas y contextos iniciales se modifiquen y por tanto, es necesario proyectar trayectorias de desarrollo profesional para los directores y equipos de gestión (PONT; NUSCHE; MOORMAN, 2008) acompañadas de políticas públicas para la implementación de acciones y estrategias en esta materia.

En segundo lugar, la necesidad de que los programas de formación y acompañamiento se centre en la enseñanza y aprendizaje de competencias. En este sentido, es importante definir el tipo de competencias que serán trabajadas, estableciendo una relación directa con los estándares de desempeño de las funciones y que permitan delimitar el tipo de práctica o hábito que debe modificarse o potenciarse (ELMORE, 2010)

En tercer lugar, el diseño de los programas de formación requieren incluir experiencias prácticas y tareas auténticas que reflejen la realidad de sus contextos educativos y se relacionan directamente al desempeño de las tareas y actividades propias de cada función, logrando así no solo modificar la práctica o el hábito, si no también, permitir la reflexión acerca del propio quehacer (LABRA et al., 2005; MONTECINOS; CORTEZ, 2015).

En cuarto lugar, la necesidad de contextualizar la experiencia de aprendizaje y permitir interacción social entre distintas realidades educativas que fomentaría la construcción de representaciones de realidades distintas y además beneficiaría la construcción de comunidades de aprendizaje y desarrollo profesional permanentes (MARCELO, 2002)

Finalmente, la necesidad de que dichos programas favorezcan estrategias de solución de problemas, que les confiera a los directores y equipos directivos, capacidades de análisis y pensamiento estratégico para apoyar la toma de decisiones y planificar líneas de mejoramiento vinculadas a la calidad de los procesos de aprendizaje de toda la comunidad educativa. 


\section{Assessoria a equipes de gestão e liderança educacional a partir da abordagem de competências funcionais}

\section{Resumo}

Este trabalho apresenta uma experiência de assessoria a equipes de gestão da região do Maule, no Chile, focado principalmente na aprendizagem de competências funcionais associadas ao desempenho das equipes de gestão. Para o efeito, foi concebido, implementado e avaliado um programa de acompanhamento socioeducativo em que participaram 11 estabelecimentos de ensino. Os resultados analisados de acordo com dois niveis de avaliação das realizações de aprendizagem mostraram um alto nível de satisfação dos participantes, bem como a aprendizagem relacionada às etapas para resolver problemas

Palavras-chave: Liderança Educacional. Programa assessoramento. Competências funcionais.

\section{Advice to management teams and educational leadership from the approach of functional competencies}

\section{Abstract}

This paper presents an experience of advising management teams from the Maule region, Chile, focused mainly on the learning of functional competencies associated to the performance of management teams. For this, a program of socio-educational support was designed, executed and evaluated in which 11 educational establishments participated. Results analyzed according to two levels of evaluation of learning achievements showed a high level of satisfaction of the participants, as well as learning related to the steps for solving problems.

Keywords: Educational leadership. Program counseling. Functional competences. 


\section{Referencias}

BOLÍVAR, A. El liderazgo educativo y su papel en la mejora: una revisión actual de sus posibilidades y limitaciones. Psicoperspectivas, v. 9, n. 2, p. 9-33, jul./dic. 2010. https://doi.org/10.5027/psicoperspectivas-Vol9-Issue2-fulltext-112

BORKO, H. Professional development and teacher learning: mapping the terrain. Educational Researcher, v. 33, n. 8, p. 3-15, Nov. 2004.

CANCINO, V.; VERA, L. Políticas educativas de fortalecimiento del liderazgo directivo: desafíos para Chile en un análisis comparado con países OCDE. Ensaio: Avaliação e Políticas Públicas em Educação, v. 25, n. 94, p. 1-33, jan./mar. 2017. https://doi.org/10.1590/s0104-40362017000100002

CHILE. Ministerio de Educación. Ley 20.501, de 8 de febrero de 2011.Calidad y equidad de la educación. Disponible en: $<$ https://www.leychile.cl/Navegar?i $\mathrm{dNorma}=1022346 \&$ buscar $=$ Ley $+20.501>$. Acceso en: 12 dic. 2016.

. Ministerio de Educación. Marco para la buena dirección y el liderazgo escolar. Santiago: Centro de Perfeccionamiento, Experimentación e Investigaciones Pedagógicas, 2015.

. Ministerio de Educación. Objetivos del plan de formación de directores de excelencia. Santiago, 2013. Disponible em: $<\mathrm{http}: / / \mathrm{www}$. formaciondirectores.mineduc.cl>. Acceso em: 20 mar. 2016.

DARLING-HAMMOND, L. El derecho de aprender: crear buenas escuelas para todos. Barcelona: Ariel, 2001.

ELMORE, R. Mejorando la escuela desde la sala de clases. Santiago de Chile: Fundación Chile, 2010.

LABRA, P. et al. La investigación- acción como herramienta para lograr coherencia de acción en el proceso de práctica profesional durante la formación inicial docente. Estudios Pedagógicos, v. 31, n. 2, p. 137-43, 2005. https://doi.org/10.4067/S0718-07052005000200009

LEITHWOOD, K. ¿Cómo liderar nuestras escuelas? Aportes desde la investigación. Santiago de Chile: Salesianos, 2009.

LEITHWOOD, K. et al. Successful school leadership: what it is and how it influences pupil learning. United Kingdom: National College for School Leadership, 2006. 
LEITHWOOD, K.; HARRIS, A.; HOPKINS, D. Seven strong claims about successful school leadership. School Leadership and Management, v. 28, n. 1, p. 27-42, 2008 .

LEITHWOOD, K.; STAGER, M. Expertise in principals' problem solving. Educational Administration Quarterly, v. 25, n. 2, p. 126-61, 1989. https://doi. org/10.1177/0013161X89025002003

LINCOLN, Y.; GUBA, E. Naturalisti inquiry. Newbury Park: Sage Publications, 1985.

MARCELO, C. Aprender a enseñar para la sociedad del conocimiento. Education Policy Analysis Archives, v. 10, n. 35, p. 1-52, ago. 2002.

MONTECINOS, C.; CORTEZ, M. Experiencias de desarrollo y aprendizaje profesional entre pares en Chile: implicaciones para el diseño de una política de desarrollo docente. Docencia, n. 55, p. 52-61, mar. 2015.

MUÑOZ STUARDO, G.; MARFÁN SÁNCHEZ, J. Competencias y formación para un liderazgo escolar efectivo en Chile. Pensamiento Educativo. Revista de Investigación Educacional Latinoamericana, v. 48, n. 1, p. 63-80, 2011.

NATIONAL INSTITUTE OF STANDARDS AND TECHNOLOGY (NIST). Malcolm Balbridge: award criteria. Gaithersburg, 1995.

PONT, B.; NUSCHE, D.; MOORMAN, H. Mejorando el liderazgo escolar. Paris: Organización para la Cooperación y el Desarrollo Económico, 2008. Vol. 1: Política y práctica,

PRASLOVA, L. Adaptation of Kirkpatrick's four level model of training criteria to assessment of learning outcomes and program evaluation in higher education. Educational Assessment Evaluation and Accountability, vol. 22, n. 3, p. 215-25, Aug. 2010. https://doi.org/10.1007/s11092-010-9098-7

PUTNAM, R.; BORKO, H. What do new views of knowledge and thinking have to say about research on teacher learning? Educational Researcher, v. 29, n. 1, p. 4-15, jan./feb. 2000. https://doi.org/10.2307/1176586

RUIZ OLABUÉNAGA, J. I. Teoría y práctica de la investigación cualitativa. Bilbao: Universidad de Deusto, 2012. 
URIBE, M. Profesionalizar la dirección escolar potenciando el liderazgo: una clave ineludible en la mejora escolar. Desarrollo de perfiles de competencias directivas en el sistema educativo chileno. Revista Iberoamericana de Evaluación Educativa, v. 3, n. 1e, p. 304-22, 2010.

WEINSTEIN, J. Liderazgo directivo, asignatura pendiente de la reforma educacional chilena. Estudios Sociales, n. 117, p. 123-47, 2009.

\section{Informações dos autores}

Carolina Iturra Herrera: Doctor en Psicología Educacional, Universidad de Salamanca. Profesor Asociado, Facultad de Psicología, Universidad de Talca. Investigador del Centro de Investigación en Ciencias Cognitivas, Universidad de Talca. Académica de Postgrado en la Universidad de Talca. Contato: citurra@utalca.cl

Víctor Eduardo Cancino Cancino: Doctorando en Dirección de Empresas, UPC Barcelona. MBA, U. de Chile. Profesor Asociado, Universidad Santo Tomás Chile. Investigador del Centro de Investigación y Modelación en Negocios. Académico de Postgrado en la Universidad de Talca, Universidad Santo Tomás y Universidad Autónoma de Nueva León. Contato: vcancino@santotomas.cl 